\title{
Case Report: Ruptured Middle Cerebral Artery Aneurysm With Intrasylvian Hematoma Successfully Treated by Coil Embolization and Minimally Invasive Puncture and Drainage
}

OPEN ACCESS

Edited by:

Xinjian Yang,

Capital Medical University, China

Reviewed by:

Zhongbin Tian,

Southern Medical University, China

Ying Zhang,

Capital Medical University, China

*Correspondence:

Limin Zheng

zlm101010@163.com

Guojun Wang

wguojun2002@163.com

tThese authors have contributed equally to this work and share first authorship

¥These authors have contributed equally to this work

Specialty section:

This article was submitted to Endovascular and Interventional Neurology,

a section of the journal

Frontiers in Neurology

Received: 28 December 2020

Accepted: 20 May 2021

Published: 21 June 2021

Citation:

Li Z, Hu Q, Zhao L, Huang H,

Zhang S, Zheng L and Wang G (2021) Case Report: Ruptured Middle Cerebral Artery Aneurysm With Intrasylvian Hematoma Successfully Treated by Coil Embolization and Minimally Invasive Puncture and Drainage. Front. Neurol. 12:646990. doi: 10.3389/fneur.2021.646990

\section{Zhen $\mathrm{Li}^{1 \dagger}$, Quan Hu${ }^{1 \dagger}$, Li Zhao ${ }^{2}$, Huayun Huang ${ }^{1}$, Shizhong Zhang ${ }^{1}$, Limin Zheng ${ }^{1 * \neq}$ and Guojun Wang ${ }^{1 * \pm}$}

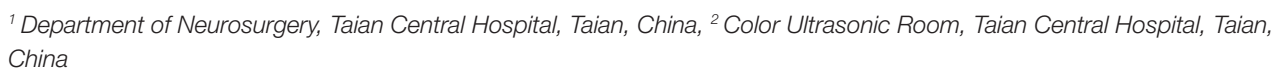

Up to one-third (12-35\%) of patients with aneurysmal subarachnoid hemorrhage experience intracerebral hematoma. Ruptured middle cerebral artery (MCA) aneurysm with hematoma is usually accompanied by progressive cerebral swelling with poor outcomes; however, it can be successfully treated by coil embolization and minimally invasive puncture and drainage. From February 2012 to March 2019, six surgeries for ruptured MCA aneurysms with intrasylvian hematoma were performed at our clinic. All patients had intracranial hematomas of $<30 \mathrm{ml}$ and GCS scores $>8$. The patients were treated by coil embolization and minimally invasive puncture and drainage. The aneurysms in all patients were completely embolized and the hematomas were mostly removed by minimally invasive puncture. The Glasgow outcome scale (GOS) scores of all patients were more than 4 at discharge when they discharged. Coil embolization and minimally invasive puncture and drainage are viable treatments for ruptured MCA aneurysms with hematomas, especially if the patient is too old, in a complicated state to undergo craniotomy, is unwilling to undergo craniotomy, or is at a greater risk of bleeding 3 days after surgery.

Keywords: ruptured middle cerebral artery aneurysm, hematoma, coil embolization, minimally invasive puncture and drainage, outcomes

\section{INTRODUCTION}

Intracerebral hematoma (ICH) due to rupture of intracranial aneurysm (IA) occurs in 10-38\% of cases with subarachnoid hemorrhage (SAH) (1). Aneurysmal ICH complicates the natural course of the disease and is associated with increased morbidity and mortality (2). Middle cerebral artery (MCA) aneurysms are most likely to result in an intracerebral and intrasylvian hematoma after their rupture $(3,4)$. About $30-37.5 \%$ of ruptured middle cerebral artery aneurysms can lead to intracranial hematoma. Most neurosurgeons prefer microsurgical clipping for treatment of such patients. Smith et al., through a systematic review and meta-analysis, recommended surgical clipping for unruptured MCA aneurysms (5). Along with the publication of International 
TABLE 1 | Characteristics of six patients with middle cerebral artery aneurysms.

\begin{tabular}{llcccccc}
\hline & Sex & Age & GCS & Hunt-hess & GOS & Antiplatelet & Stent \\
\hline Case 1 & Male & 42 & 11 & 3 & 5 & NO & NO \\
Case 2 & Male & 48 & 9 & 4 & 4 & NO & NO \\
Case 3 & Female & 69 & 10 & 2 & 5 & YES & YES \\
Case 4 & Female & 44 & 12 & 2 & 5 & NO & NO \\
Case 5 & Female & 76 & 10 & 3 & 4 & NO & NO \\
Case 6 & Male & 50 & 13 & 2 & 5 & YES & YES \\
\hline
\end{tabular}

GCS, Glasgow coma scale; GOS, Glasgow outcome scale.

Subarachnoid Aneurysm Trial (ISAT) and the improvement of neurointervention techniques, more neurosurgical centers have been opting for endovascular coiling $(6,7)$. However, for ruptured MCA aneurysms with hematomas, most patients still prefer craniotomy clipping. In China, some scholars believe that endovascular coiling is effective in patients with ruptured MCAs (8). Whether interventional embolization can be performed for ruptured middle cerebral artery aneurysms with hematoma is still uncertain. We have reported six cases with ruptured MCA aneurysm and intrasylvian hematoma that were successfully treated by coil embolization and minimally invasive puncture and drainage.

\section{SUBJECTS AND METHODS}

\section{Patient Population}

From February 2012 to March 2019, six patients (total of six MCA aneurysms) underwent endovascular coiling and minimally invasive puncture and drainage in our institution. The clinical condition at admission was classified according to the Hunt-Hess grade. Post-operative CT scans were obtained for all patients, and the hematoma volume was calculated using the formula $\mathrm{a} \times \mathrm{b} \times$ $c / 2$. The clinical outcomes were graded according to the Glasgow Outcome Scale (GOS). MCA aneurysms were confirmed by DSA and treated by an endovascular approach. All the intracranial hematomas were confirmed by CT and treated by minimally invasive puncture and drainage (Table 1).

The outcomes of patients were evaluated by GOS. Patients with GOS score 1-3 were defined as having poor outcomes and those with GOS scores 4-5 were defined as having good outcomes. Informed consent was obtained from patients and the study was approved by our institutional review board.

\section{RESULTS}

\section{Baseline Characteristics}

The clinical characteristics and treatment details of the six patients are shown in Table 1. The sample comprised two men and four women with a mean age of 58.7 (range 42-76) years. All patients had a GLS score $>8$ before the surgery. The GOS scores of all patients were more than 4 at discharge.

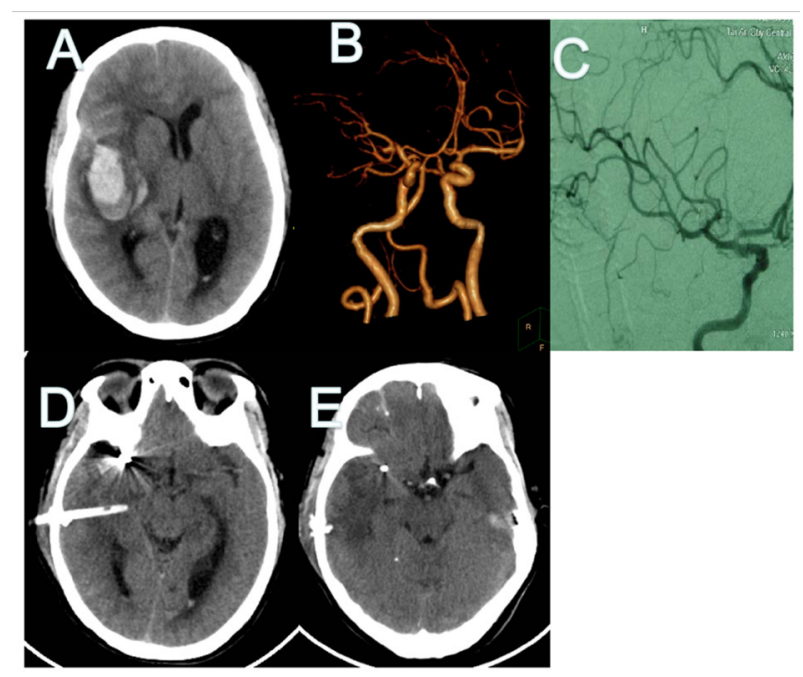

FIGURE 1 | CT and CT angiography in case 1. (A,B) Initial CT scans reveal characteristic findings of ruptured MCA aneurysm with intrasylvian hematoma. Hematoma volume was calculated as $28.8 \mathrm{ml}$. (C) Post-operative angiography shows the aneurysm is completely occluded and parent artery is unimpeded. (D) Immediate post-operative CT finding the drainage tube was in good position. (E) CT finding Hematoma's almost drained clean on POD 2. CT, computed tomography; MCA, middle cerebral artery; POD, post-operative day.

\section{Illustrative Cases Case One}

A 48-year-old man presented to the emergency room with a severe headache after sudden loss of consciousness. Initial consciousness level was deep drowsy (Hunt-Hess 4). Brain CT showed a typical SAH from MCA aneurysmal rupture, in which the SAH was mainly dispersed prominently along the left Sylvian fissure. Hematoma volume was calculated as $28.7 \mathrm{ml}$ using the formula $\mathrm{a} \times \mathrm{b} \times \mathrm{c} / 2$ (Figures 1A,B). Coil embolization surgery was performed $6 \mathrm{~h}$ after admission. Minimally invasive puncture and drainage were performed immediately after the operation. Surgery was performed successfully on the ruptured aneurysm and no complication was observed during the procedure (Figures 1C,D). On the two day after the operation, CT showed that the hematoma had disappeared (Figure 1E).

\section{Case Two}

A 42-year-old man was transferred to our hospital 3 days after the onset of symptoms (a severe headache) (Hunt-Hess 2). Brain $\mathrm{CT}$ and $\mathrm{CT}$ angiography revealed $\mathrm{SAH}$ from ruptured MCA aneurysm and large amounts of intrasylvian hematoma (about $16.5 \mathrm{ml}$; Figures 2A,B). Coil embolization surgery was performed $5 \mathrm{~h}$ after admission. Minimally invasive puncture and drainage were performed immediately after the operation. Surgery was performed successfully on the ruptured aneurysm and there was no complication during the procedure (Figures 2C,D). On the third day after the operation, CT showed that the hematoma had almost disappeared (Figure 2E). 


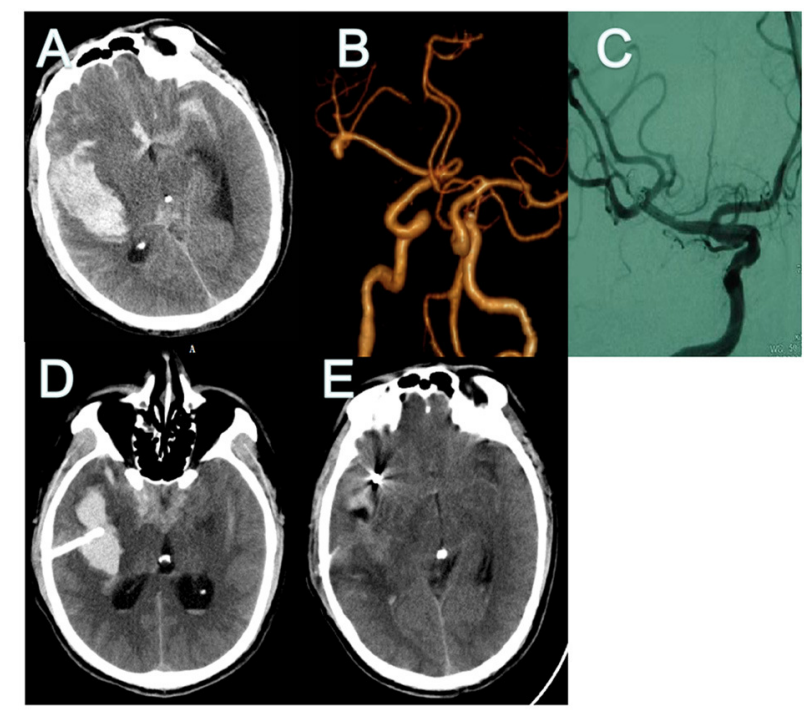

FIGURE 2 | CT and CT angiography in case 1. (A,B) Initial CT scans reveal characteristic findings of ruptured MCA aneurysm with intrasylvian hematoma. Hematoma volume was calculated as $16.5 \mathrm{ml}$. (C) Post-operative angiography shows the aneurysm is completely occluded and parent artery is unimpeded. (D) Immediate post-operative CT finding the drainage tube was in good position. (E) CT finding Hematoma's almost drained clean on POD 3. CT, computed tomography; MCA, middle cerebral artery; POD, post-operative day.

\section{DISCUSSION}

The MCA has a complex anatomical structure, which contains multiple ventral perforating arteries. It is widely distributed, with branches lacking collagen circulation. Ruptured aneurysms in the brain can lead to neurological disorders, such as hemiplegia, aphasia, and paresthesia. MCA aneurysms account for $20 \%$ of intracranial aneurysms (1). Ruptured intracranial aneurysms are often accompanied by large intracranial hematomas, as compared to other intracranial aneurysms. Hence, the HuntHess grade is worse in such cases. About $30 \%$ of ruptured middle cerebral artery aneurysms can lead to intracranial hematoma (7). Zhao et al. reported that $37.3 \%$ of the patients had intracranial hematomas, and that inappropriate treatment may affect branch blood flow causing chemical syndrome or cerebral infarction (8).

Currently, the treatment methods for ruptured aneurysms mainly include microsurgical clipping and endovascular embolization. With the publication of ISAT, more neurosurgical centers begun to choose endovascular embolization. However, they have not elaborated on the best treatment for aneurysms, which remains controversial. The complex anatomical structure of the MCA, such as bifurcation and multiple branches, increases the difficulty of performing an interventional surgery. For ruptured wide-necked aneurysms, early stent placement can lead to thrombosis $(5,9)$. However, for some patients who are not suitable for craniotomy (such as old age, long-term anticoagulant therapy, etc.) there is still no good treatment plan.
With the improvement of interventional techniques and the application of new interventional materials, endovascular embolization techniques, especially three-dimensional rotary angiography, and stent-assisted embolization, can be used to treat more complex large aneurysms. Three-dimensional rotational angiography can clearly show the anatomical features of the MCA and its branches. Stenting reduces the recurrence rate of MCA aneurysms by changing the cerebral hemodynamics and endothelialization of the stent. The effect of stent network on the parent artery branch remains minor. Many studies have confirmed the safety and efficacy of endovascular embolization and have obtained comparable results to that of surgical clipping $(2,5,10,11)$. At present, most scholars still believe that microsurgical clipping should be the preferred treatment for ruptured MCA aneurysms with hematoma. Our surgical experience was that the brain tissue was obviously swollen and the lateral fissure was difficult to separate after 3 days of subarachnoid hemorrhage. We reported six patients with ruptured MCA aneurysms with intrasylvian hematoma who were treated in our clinic. All patients had intracranial hematomas of $<30 \mathrm{ml}$ and GCS scores $>8$. The patients were treated by coil embolization and minimally invasive puncture and drainage. Good prognosis was obtained. However, microsurgical clipping remains to be the preferred treatment for patients with massive hematomas and cerebral hernia.

There were some limitations in this study. This was not a randomized study, and the number of patients and the duration of follow-up were limited. Due to the small sample and lack of randomized controlled studies, our retrospective study lacked the support of a statistical analysis, However, this paper is only published as a case report and only provides a treatment idea. For the advantages and disadvantages of this approach, a lot of comprehensive analysis of clinical cases is needed, which is our work in the future.

\section{CONCLUSION}

Coil embolization and minimally invasive puncture and drainage are viable treatments for ruptured MCA aneurysms with hematomas, especially if the patient is too old, in a complicated condition to undergo craniotomy, is unwilling to undergo craniotomy, or is at a greater risk of bleeding 3 days after surgery. In addition, for some young women or special working population, the future work might be affected by surgical scar.

\section{DATA AVAILABILITY STATEMENT}

The original contributions presented in the study are included in the article/supplementary material, further inquiries can be directed to the corresponding author/s.

\section{ETHICS STATEMENT}

The studies involving human participants were reviewed and approved by the ethics committee of Taian Central Hospital. The 
patients/participants provided their written informed consent to participate in this study. Written informed consent was obtained from the individual(s) for the publication of any potentially identifiable images or data included in this article.

\section{AUTHOR CONTRIBUTIONS}

All authors listed have made a substantial, direct and intellectual contribution to the work, and approved it for publication.

\section{REFERENCES}

1. Abbed KM, Ogilvy CS. Intracerebral hematoma from aneurysm rupture. Neurosurg Focus. (2003) 15E4. doi: 10.3171/foc.2003.15.4.4

2. Pasqualin A, Bazzan A, Cavazzani P, Scienza R, Licata C, Da Pian R. Intracranial hematomas following aneurysmal rupture: experience with 309 cases. Surg Neurol. (1986) 25:6-17. doi: 10.1016/0090-3019(86)90107-2

3. Sehba FA, Hou J, Pluta RM, Zhang JH. The importance of early brain injury after subarachnoid hemorrhage. Prog Neurobiol. (2012) 97:1437. doi: 10.1016/j.pneurobio.2012.02.003

4. Nowak G, Schwachenwald D, Schwachenwald R, Kehler U, Müller H, Arnold H. Intracerebral hematomas caused by aneurysm rupture. Experience with 67 cases. Neurosurg Rev. (1998) 21:5-9. doi: 10.1007/BF01111478

5. Smith TR, Cote DJ, Dasenbrock HH, Hamade YJ, Zammar SG, El Tecle NE, et al. Comparison of the efficacy and safety of endovascular coiling versus microsurgical clipping for unruptured middle cerebral artery aneurysms: a systematic review and metaanalysis. World Neurosurg. (2015) 84:942-53. doi: 10.1016/j.wneu.2015.0 5.073

6. Güresir E, Beck J, Vatter H, Setzer $M$, Gerlach R, Seifert V, et al. Subarachnoid hemorrhage and intracerebral hematoma: incidence, prognostic factors, and outcome. Neurosurgery. (2008) 63:1088-93. doi: 10.1227/01.NEU.0000335170.76722.B9

7. Eboli P, Ryan RW, Alexander JE, Alexander MJ. Evolving role of endovascular treatment for MCA bifurcation aneurysms: case series of 184 aneurysms and review of the literature. Neurol Res. (2014) 36:332-8. doi: 10.1179/1743132814Y.000000 0324

\section{FUNDING}

This study was supported by the Natural Science Foundation of Shandong Province of China, No. ZR2020MH157 (to QH).

\section{ACKNOWLEDGMENTS}

We would like to thank Editage (www.editage.com) for English language editing.

8. Zhao X, Li Z, Fang X, Liu J, Wu D, Lai N. Treatment of ruptured middle cerebral artery aneurysms by endovascular approach: a single-center experience. Int $J$ Neurosci. (2017) 127:433-8. doi: 10.1080/00207454.2016.1190923

9. Kadkhodayan Y, Delgado Almandoz JE, Fease JL, Scholz JM, Blem AM, Tran $\mathrm{K}$, et al. Endovascular treatment of 346 middle cerebral artery aneurysms: results of a 16-year single-center experience. Neurosurgery. (2015) 76:5460. doi: 10.1227/NEU.0000000000000562

10. Diaz OM, Rangel-Castilla L, Barber S, Mayo RC, Klucznik R, Zhang YJ. Middle cerebral artery aneurysms: a single-center series comparing endovascular and surgical treatment. World Neurosurg. (2014) 81:3229. doi: 10.1016/j.wneu.2012.12.011

11. Geyik S, Yavuz K, Yurttutan N, Saatci I, Cekirge HS. Stent-assisted coiling in endovascular treatment of 500 consecutive cerebral aneurysms with long-term follow-up. AJNR Am J Neuroradiol. (2013) 34:215762. doi: 10.3174/ajnr.A3574

Conflict of Interest: The authors declare that the research was conducted in the absence of any commercial or financial relationships that could be construed as a potential conflict of interest.

Copyright (c) $2021 \mathrm{Li}, \mathrm{Hu}$, Zhao, Huang, Zhang, Zheng and Wang. This is an openaccess article distributed under the terms of the Creative Commons Attribution License (CC BY). The use, distribution or reproduction in other forums is permitted, provided the original author(s) and the copyright owner(s) are credited and that the original publication in this journal is cited, in accordance with accepted academic practice. No use, distribution or reproduction is permitted which does not comply with these terms. 